Communication

\title{
The PIP Peptide of INFLORESCENCE DEFICIENT IN ABSCISSION Enhances Populus Leaf and Elaeis guineensis Fruit Abscission
}

\author{
Timothy John Tranbarger ${ }^{1,2, *(\mathbb{C}, \text {, Hubert Domonhédo }}{ }^{3}$, Michel Cazemajor ${ }^{3,4}$, Carole Dubreuil ${ }^{5,6}$, \\ Urs Fischer $^{5,7}$ and Fabienne Morcillo ${ }^{8}$ \\ 1 UMR DIADE, Institut de Recherche pour le Développement, Université de Montpellier, \\ 34394 Montpellier, France \\ 2 Ecology and Genetics Laboratory, Pontificia Universidad Católica del Ecuador (PUCE), \\ 17-01-21-84 Quito, Ecuador \\ 3 CRAPP, INRAB, BP 1 Pobè, Benin; hubertdomonhedo@yahoo.fr (H.D.); \\ Michel.Cazemajor@palmelit.com (M.C.) \\ 4 PalmElit SAS, F-34980 Montferrier-sur-Lez, France \\ 5 Department of Forest Genetics and Plant Physiology, Umeå Plant Science Centre, \\ Swedish University of Agricultural Sciences, SE-901 83 Umeå, Sweden; Carole.DUBREUIL@cea.fr (C.D.); \\ Urs.Fischer@kws.com (U.F.) \\ 6 DRT DPACA, CEA Tech Cadarache, 13108 Saint Paul Lez Durance, France \\ 7 KWS SAAT SE, RD-BT, 37574 Einbeck, Germany \\ 8 UMR DIADE, CIRAD, F-34398 Montpellier, France; fabienne.morcillo@ird.fr \\ * Correspondence: timothy.tranbarger@ird.fr; Tel.: +33(0)467-416-235
}

Received: 3 May 2019; Accepted: 28 May 2019; Published: 30 May 2019

check for updates

\begin{abstract}
The programmed loss of a plant organ is called abscission, which is an important cell separation process that occurs with different organs throughout the life of a plant. The use of floral organ abscission in Arabidopsis thaliana as a model has allowed greater understanding of the complexities of organ abscission, but whether the regulatory pathways are conserved throughout the plant kingdom and for all organ abscission types is unknown. One important pathway that has attracted much attention involves a peptide ligand-receptor signalling system that consists of the secreted peptide IDA (INFLORESCENCE DEFICIENT IN ABSCISSION) and at least two leucine-rich repeat (LRR) receptor-like kinases (RLK), HAESA (HAE) and HAESA-LIKE2 (HSL2). In the current study we examine the bioactive potential of IDA peptides in two different abscission processes, leaf abscission in Populus and ripe fruit abscission in oil palm, and find in both cases treatment with IDA peptides enhances cell separation and abscission of both organ types. Our results provide evidence to suggest that the IDA-HAE-HSL2 pathway is conserved and functions in these phylogenetically divergent dicot and monocot species during both leaf and fruit abscission, respectively.
\end{abstract}

Keywords: organ abscission; fruit abscission; leaf abscission; cell separation; peptide signalling; LRR-RLK; Populus; oil palm; abscission zone

\section{Introduction}

Organ abscission is a process that enables plants to shed whole organs that have completed their function or as a defence response when infected or damaged [1]. At the molecular level, organ abscission is a complex process that includes the integration of both endogenous and exogenous signals derived from the physiological and environmental status of the plant [2]. At the cellular level, organ abscission is dependent on mechanisms that cause adjacent cells to lose their adherence 
to one another and separate [1]. Furthermore, these cell separation mechanisms involve cell wall modifications, in particular the hydrolysis of pectin within the middle lamella of adjacent cells, which occur specifically with cells at the base of the organ to be shed, within a specialized tissue called the abscission zone (AZ) [1,3]. The function of the AZ allows plants to shed almost any organ, including leaves, flowers, floral parts, and fruit at the appropriate time. While these various organ types have different ontogenies, with few exceptions, the basis for organ abscission is related to the function and signalling mechanisms that occur in the AZ.

Molecular genetic research on floral organ abscission with the model species Arabidopsis thaliana has led to tremendous progress in deciphering the underlying mechanisms and to the definition of details concerning the stages of organ abscission [4-8]. While these signalling and molecular pathways identified in Arabidopsis provide insight into the stages and signalling mechanisms of organ abscission, it is not known whether this information can be translated to all species and all organ types that are shed [9]. Based on the current model derived from these studies, the four stages of abscission include: (1) the ontogeny of the $A Z,(2)$ the acquisition of $A Z$ cell competence to integrate and respond to abscission signals, (3) the activation of AZ cells by abscission signals that induce the de novo synthesis of cell wall-modifying enzymes that cause cell wall loosening and rounding of the AZ cells, and (4) the trans-differentiation of remaining AZ cells to produce a protective layer $[8,10]$.

One signalling pathway discovered in Arabidopsis, which has become a significant focus of research, involves a peptide ligand-receptor system [10-12]. The signalling pathway consists of the secreted peptide IDA (INFLORESCENCE DEFICIENT IN ABSCISSION) encoded by the gene INFLORESCENCE DEFICIENT IN ABSCISSION (At1g68765), and the two leucine-rich repeat (LRR) receptor-like kinases (RLK) HAESA (HAE) and HAESA-LIKE2 (HSL2) [12-14]. It was demonstrated that the IDA peptide binds and activates HSL2, and that the 12 amino acid long PIP peptide with hydroxylation of a central proline residue is the most efficient peptide for activation [13]. Both ida and hae hsl2 mutants retain their floral organs, while the overexpression of the IDA gene rescues the ida mutant, which reverts to the wild-type abscission phenotype, and overexpression in the wild-type of either IDA or similar IDA-LIKE (IDL) gene family members results in early abscission [11,12]. A recent survey of the literature suggests that the IDA-HAE-HSL2 pathway functions during the final stages of organ abscission (stages 3 and/or 4), and not during the stages of acquisition of competence or activation of the AZ (stages 1 and 2) [10]. However, while it is clear that the IDA-HAE-HSL2 signalling pathway functions during Arabidopsis floral organ abscission, it is not known whether this pathway functions in other species and with organs such as leaves or fruit.

Several recent studies have begun to respond to the question of whether the IDA-HAE-HSL2 pathway functions in different species and organs. For example, IDL genes identified in both citrus and litchi ectopically expressed in Arabidopsis promoted floral organ shedding, suggesting a conserved role for this pathway in both these species $[15,16]$. While these studies show conservation of function in the context of Arabidopsis, evidence of function within citrus and litchi is not available. A previous study by our groups identified similar genes that encode IDL, HAE, and HSL2 proteins from Populus and oil palm (Elaeis guineensis) with expression correlated to leaf and fruit abscission, respectively [17]. Both of these organ abscission processes are important for the production capacities of each of these species; fruit yield and the facilitation of harvest for oil palm and biomass production for poplar. While our previous studies showed that the functional components of the pathway are present, no evidence is available on the function of the pathway within these two species. In the current study, we address the question of whether the PIP peptide of IDA has the capacity to enhance these different organ abscission processes in two widely divergent species. The results with both organ abscission systems showed that small but significant increases in the abscission of both leaf and fruit occur after treatment with the PIP peptide of IDA. The results demonstrate the bioactive capacity of the PIP peptide of IDA in both these abscission systems, and suggest that the IDA-HAE-HSL2 pathway is conserved and functions in these phylogenetically divergent dicot and monocot species during both leaf and fruit abscission, respectively. 


\section{Results}

\subsection{Exogenous Treatments of PtIDA and PtIDL1 Peptides and Their Effect on Poplar Leaf Abscission}

Leaf abscission in the hybrid aspen T89 (Populus tremula X P. tremuloides) is induced by dark treatment of leaves, which has been used previously as an experimental system to analyse the abscission process at the molecular level $[17,18]$. Using the same experimental system, it was previously shown that the Populus PtIDA and PtIDL1 gene transcripts increased significantly during dark induced leaf abscission [17]. In the current experiments, this system was used to test whether either the PtIDA or PtIDL1 synthetic peptides have a bioactive effect during dark-induced leaf abscission in Populus. Our hypothesis was that these peptides might be able to enhance leaf abscission after dark treatment, which would provide further evidence that the IDA-HAE-HSL2 pathway functions during dark-induced leaf abscission of Populus. For the experiment, at the same time as the dark induction of leaf abscission $(t=0)$, leaf axils were treated with either PtIDA or PtIDL1 synthetic peptides at the same time $(t=0)$, and again at 7 days after dark induction (DAD). After 1 day of the second peptide treatment (8 DAD), $10 \%$ more leaves shed from plants treated with either PtIDA or PtIDL1 (Figure 1a). A higher percentage of leaf abscission continued to be observed under both peptide treatments up until 12 DAD, while no increase in percentage of leaves shed was observed at the remaining time points in the PtIDL1 peptide-treated plants. In contrast, the PtIDA peptide continued to enhance the percentage of leaf abscission up to $20 \%$ from 14 DAD to 18 DAD. The abscission enhancing effect of the PtIDA peptide decreased at $20 \mathrm{DAD}$, and by $22 \mathrm{DAD}$ there was no difference in the percentage of leaves lost between the controls and the peptide-treated plants. Furthermore, plants treated with the PtIDA peptide lost their leaves significantly earlier than the control or PtIDL1-treated plants (Figure 1b). The results indicate that both the PtIDA and the PtIDL1 peptides can enhance dark-induced leaf abscission of Populus, but their effects are not the same, with the PtIDA peptide being more efficient than the PtIDL1 peptide.

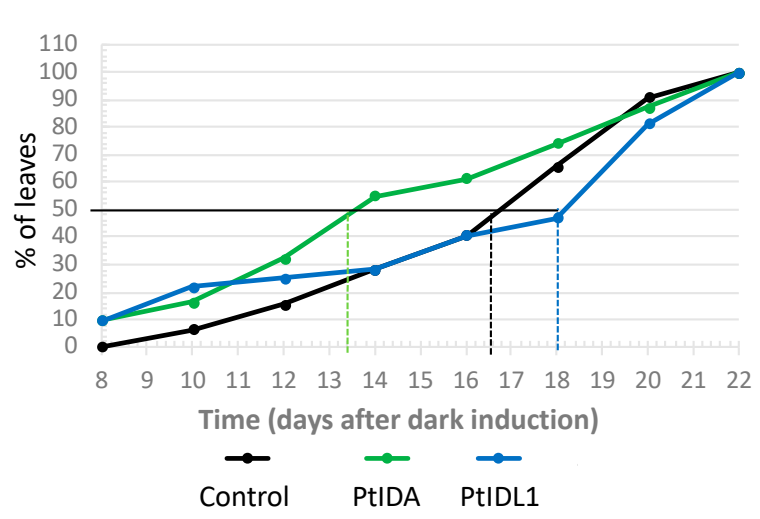

(a)

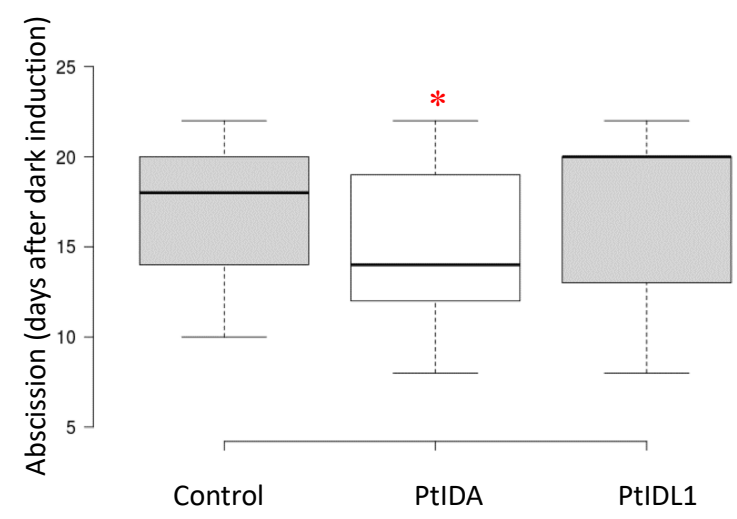

(b)

Figure 1. Poplar leaf abscission induced by dark treatment is enhanced in response to PtIDA or PtIDL1 peptide treatments of leaf axils. (a) Time course of treatment with $0.1 \mu \mathrm{M}$ PtIDA, PtIDL1, or water (control) 7 days after dark induced abscission. The percentage of dark-induced leaves dropped. $\mathrm{n}=31$ leaves for PtIDA and $\mathrm{n}=32$ for control treatment (water) and PtIDL1; dotted lines represent timepoints when $50 \%$ of the leaves were dropped. (b) PtIDA-treated leaves separated significantly earlier $\left({ }^{*}, \mathrm{t}\right.$-test; $\mathrm{n}=31$ or 32 leaves; $\mathrm{p}<0.05$ ) than mock-treated leaves. Boxplot: central lines represent the median, limits of boxes are the 25 th and 75 th percentiles, and whiskers are the $1.5 \times$ interquartiles.

\subsection{Exogenous Treatments of the EgIDA Peptide and Effects on Cell Separation in the Oil Palm Fruit AZ}

Fruit abscission of oil palm occurs naturally with ripe fruit in the field and is induced by ethylene [19-22]. In a previous study, it was shown that at least two IDL transcripts (EgIDA2 and 
EgIDA5) were expressed in the ripe fruit $\mathrm{AZ}$, and this expression was enhanced by ethylene [17]. We developed an in vitro phenotype test to examine the abscission behaviour and to screen for individuals of oil palm that have delayed abscission [23,24]. We used this experimental system to examine the effect of the EgIDA5 peptide on oil palm ripe fruit abscission. Our hypothesis was that the synthetic EgIDA5 peptide could enhance the cell separation process of the ripe fruit AZ, which would provide further evidence that the IDA-HAE-HSL2 pathway functions during oil palm ripe fruit abscission. In previous experiments, it was found that ethylene induced fruit abscission, but the effect was more pronounced with ripe fruit [21]. In the current experiment, fruit from bunches at two different stages of development, approximately 120 and 150 days after pollination (DAP), were used to examine the effect of the synthetic EgIDA peptide on cell separation in the AZ. For these experiments, an abscission index (AI) was calculated based on four different phenotypic responses observed as described in the Materials and Methods. For the first biological repetition, AI variation depended significantly on the EgIDA peptide treatment $(p=0.000036)$, the age of the fruit $(p=0.00000)$, and the EgIDA $\times$ age of the fruit interaction $(p=0.000077)$. The EgIDA peptide had a positive effect on the AI at either concentration used. However, the effect of the peptide differed depending on the age of the fruit (Figure 2a). This indicated that the EgIDA peptide only had a significant effect on cell separation (more positive AI) of fruit at 150 DAP. These results were confirmed in a second biological repetition (Figure 2b). It was concluded that the EgIDA5 peptide enhanced abscission in ripe fruit, at a time when ethylene is being produced, but not in fruit at stages of development when ethylene production is low [25].

\section{Repetition 1}

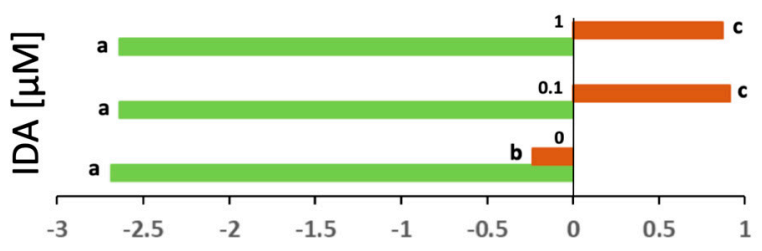

Al (Abscission Index)

\section{Repetition 2}

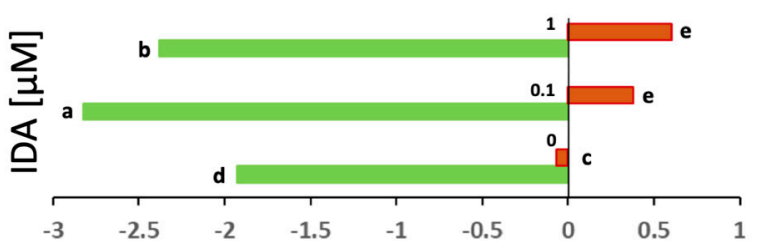

Al (Abscission Index)

120 DAP (Low ethylene)

150 DAP (High ethylene)

(a)

(b)

Figure 2. Cell separation in the oil palm fruit abscission zone (AZ) is enhanced significantly by treatments with the EgIDA peptide at concentrations of 0.1 or $1 \mu \mathrm{M}$, and the effect is only observed with ripe fruit. (a) There is a significant $(\mathrm{p}=0.000036)$ effect of the EgIDA peptide on the abscission index $(\mathrm{AI})$, and the effect is age-dependent $(\mathrm{p}=0.00000)$. (b) A second repetition confirmed the significant $($ EgIDA $\times$ age of the fruit interaction, $\mathrm{p}=0.000824)$ effect of the EgIDA peptide to enhance separation in AZs of ripe fruit (150 days after pollination (DAP)) when ethylene is produced, and not at an earlier stage of development (120 DAP) when ethylene production is low. Different lower-case letters represent statistically significant differences. Negative AI means less abscission, positive AI more abscission. The AI was calculated as described previously [24].

\section{Discussion}

The IDA-HAE-HSL2 organ abscission regulatory pathway began to be elucidated in transgenic antisense plants with a reduction of function of HAE, an LRR-RLK, in Arabidopsis [26]. Reduction of HAESE function resulted in a delay of floral organ abscission, with the severity of the phenotype correlated to the amount of HAE protein present. The results suggest that HAE may bind to an as yet unidentified ligand. The next piece of the puzzle came with the identification of ethylene-sensitive 
ida mutants that have a non-shedding floral organ phenotype [11]. The fact that IDA is a gene that encodes a small protein suggests it could be processed post-translationally to give rise to a peptide ligand recognized by a receptor, which together may act in signalling to control floral organ abscission in Arabidopsis. A 12 amino acid conserved motif named PIP in the C-terminal region of the IDA protein was thought to be important for ligand receptor function [11]. Further evidence came from a series of experiments that provided indirect evidence that HAE is the receptor for IDA [12]. That study demonstrated that the functional part of the IDA protein is found in the EPIP peptide, and that when flowers were exposed to $10 \mu \mathrm{M}$ of the EPIP peptide they promoted up to $20 \%$ more floral organ abscission than untreated flowers. It was then shown that the dodecamer PIP peptide at nanomolar concentrations is able to activate HSL2 in transient tobacco cell assays [13]. Finally, studies revealed that the PIP peptide binds to a hormone-binding pocket in the receptor HAE, and that a central hydroxyproline residue in the PIP peptide anchors IDA to the receptor [27].

In the present study, we addressed whether the functional portion of IDA peptides, the PIP motif, could be bioactive to enhance the organ abscission processes of two widely divergent species, including leaf abscission of Populus and fruit abscission of oil palm. In both experimental systems used, the results indicate that the IDA peptides are bioactive and capable of enhancing both leaf and ripe fruit abscission in Populus and oil palm, respectively. Previous reports with citrus and litchi have provided evidence for the conservation of the IDA-HAE-HSL2 pathway in these species $[15,16]$. In citrus, overexpression of the citrus IDA gene CitIDA3 in Arabidopsis results in early floral organ abscission and an increased AZ size, similar to the phenotype observed when the Arabidopsis IDA gene AtIDA is overexpressed in Arabidopsis [15,28]. In addition, overexpression of CitIDA3 in the ida-2 mutant rescues the abscission defect in these plants, providing further evidence of conservation of function [15]. With litchi, overexpression of the IDL gene LcIDL1 in Arabidopsis also results in premature floral abscission [16]. As with citrus, the overexpression of the IDL gene LcIDL1 in the ida-2 mutant results in reversion to wild-type-like floral organ abscission.

While both of these studies provided evidence of functional conservation using the Arabidopsis floral organ abscission system as a platform, neither of these studies addressed whether the IDA peptides have bioactivity to promote abscission, and whether the IDA-HAE-HSL2 pathway functions during organ abscission in either of these plant species. In addition, a recent study with Lupinus luteus L. revealed that exogenous treatment with EPIP peptides significantly increased the rate of flower abortion, which suggests that the IDA-HAE-HSL2 pathway functions in that system [29]. In the current study, we showed that the bioactivity of IDA peptides was sufficient to enhance both leaf and fruit abscission in Populus and oil palm, respectively. In addition, the experiments showed that a low concentration $(0.1 \mu \mathrm{M})$ of peptide was sufficient for abscission-promoting bioactivity, similar to the effect of Arabidopsis IDA PIP observed in activation and binding studies [13]. The results suggest that the PIP peptide of IDA has the capacity to function as a peptide hormone in planta, and provides evidence that the IDA-HAE-HSL2 pathway functions in these two diverse organ abscission systems.

Several studies have revealed that exogenous ethylene treatments induce oil palm fruit abscission, but the enhanced effect is observed more clearly with ripe fruit $[21,22,30]$. In addition, at least two $E g I D A$ transcripts (EgIDA2 and $E g I D A 5)$ are expressed in the AZ during ripe fruit abscission of oil palm, and this expression is enhanced by ethylene [17]. In the current study, we found that the enhancing effect of the EgIDA peptide depended on the stage of development, with cell separation in the ripe fruit $\mathrm{AZ}$ being more sensitive to the treatments. In a recent literature review that addressed the position of the IDA-HAE-HSL2 pathway in the regulation of floral organ abscission in Arabidopsis, it was concluded that the pathway is downstream of ethylene and functions at the last stages of the abscission process [10]. Our data support this view given that the EgIDA peptide only enhances abscission of ripe fruit, at stages when ethylene is being produced in the fruit mesocarp [25].

Remarkably, despite the phylogenetic divergence of the two species and Arabidopsis in which the IDA-HAE-HSL2 pathway was discovered, and the difference in the organ abscission processes examined, including shade-induced leaf abscission of poplar, ripe fruit abscission of oil palm, and 
floral organ abscission of Arabidopsis, the PIP peptide of IDA enhanced cell separation in all systems. Our results suggest that these peptides, as well as the amino acids in the receptors that interact with the ligand peptide, according to the structure of IDA with HAE, are extremely well conserved.

\section{Materials and Methods}

\subsection{Plant Material}

Oil palm individuals were cultivated at the Centre de Recherches Agricoles - Plantes Pérennes (CRA-PP), Station de Pobè, Bénin and derived from a self-pollinated individual (DA115D) that originated from the Deli population. Eight to 12 fruit spikelets were removed from the upper middle section of each fruit bunch and used for the in vitro abscission test described below. The date of pollination was recorded in the field and used to define the developmental stages based on days after pollination (DAP) to select fruit material for the experiments.

For dark induction experiments, the hybrid aspen clone T89, Populus tremula L. X P. tremuloides Michx, was employed. Saplings were propagated in vitro before transfer to soil and grown in a glasshouse, as described in detail in [18].

\subsection{In Vitro Phenotype Tests of Oil Palm Fruit Abscission}

The abscission index (AI) was calculated from the absolute values obtained by an in vitro phenotype test previously described with modifications [24]. From the fruit spikelets collected as described above, approximately 24 similarly ripe fruit were selected and the base of each fruit containing the $\mathrm{AZ}$ was then sliced longitudinally to give at least two slices approximately $1-1.5 \mathrm{~mm}$ in length (Figure S1). This allowed each slice to contain both the large primary AZ and the adjacent AZs previously described [19,22]. A total of 45 fruit base slices from each bunch were collected and placed on Petri plates (15 slices per plate) with dampened Whatman filter paper supplemented with $2 \mathrm{~mL}$ of EgIDA5 synthetic peptide, which consists of the PIP motif (PIPPSGPSKRHN) synthesized and quantified as previously described [13,17], in solution at concentrations of $0,0.1$, and $1 \mu \mathrm{M}$. In addition, $30 \mu \mathrm{L}$ of EgIDA5 peptide solution was deposited on the AZ of each slice (Figure S1). The fruit bases containing the $\mathrm{AZ}$ were then incubated at room temperature for $24 \mathrm{~h}$. For the phenotype test, pressure was applied with forceps by twisting the slices to determine whether separation had occurred, and whether it occurred at both the primary and secondary AZs. For the phenotypes, four classes were defined and attributed to each fruit slice tested: A, no separation; B, partial separation in primary AZ only; $C$, extensive separation in primary $A Z$ only; and $D$, complete separation in both primary and adjacent AZs (Figure S2). To calculate the AI, we used the following formula: $\left(\mathrm{nA}^{*}-3+\mathrm{nB}^{*}-1+\mathrm{nC}^{*} 1+\right.$ $\left.n D^{*} 3\right) /(n A+n B+n C+n D)$, where $n=$ total number of observations attributed per class. This formula gave a range of AI values from -3 to 3 [24]. For the EgIDA5 peptide dose response experiments, fruit from bunches at around 120 and 150 days after pollination (DAP) were used. The effect of the EgIDA peptide and the age of fruit bunch were tested on the AI, using an analysis of variance (ANOVA) with two fixed classification criteria (IDA, age). In the event of a significant effect with more than two modes, which is the case with significant interactions, the Newman and Keul multiple comparison of means test was used [31,32].

\subsection{Dark-Induced Abscission Experiments}

To induce abscission, blades of fully expanded leaves with a petiolar angle between $75^{\circ}$ and $90^{\circ}$ from $2 \mathrm{~m}$ tall trees were covered with aluminum foil. Either $30 \mu \mathrm{L}$ of $0.1 \mu \mathrm{M}$ of the synthetic peptide PtIDA or PtIDL1, which consists of the PIP motif (PtIDA, PIPPSGoSKRHN and PtIDL1, PVPPSAoSKRHN, where "o" stands for hydroxyproline; synthesized as previously described [13]), dissolved in water was applied to leaf axils. Leaf axils were treated at day 0 (beginning of dark induction) and day 7. For each treatment, four replicate trees with eight treated leaves were used. Abscission was scored on a daily basis by counting dropped leaves after gently shaking the trees. 
Supplementary Materials: The following are available online at http://www.mdpi.com/2223-7747/8/6/143/s1, Figure S1: IDA peptide methodology, Figure S2: Phenotypes for AI calculation.

Author Contributions: Conceptualization and methodology, T.J.T., F.M., and U.F.; formal analysis, T.J.T., F.M., and U.F.; investigation, T.J.T., F.M., H.D., C.D., and U.F.; resources, H.D. and M.C.; writing-original draft preparation, T.J.T.; writing - review and editing, T.J.T. and all authors read and approved the final manuscript; visualization, T.J.T., F.M., and U.F.; supervision, T.J.T., F.M., and U.F.; project administration, T.J.T., F.M., and U.F.; funding acquisition, T.J.T., F.M., and U.F.

Funding: This research was supported by funds from PalmElit SAS/IRD/CIRAD to F.M. and T.J.T.

Acknowledgments: We would like to thank Ernest Tchohntcho for his excellent work and technical assistance, and the personnel at the Institut National de Recherche Agricole du Bénin (INRAB), Centre de Recherche Agricoles Plantes Pérennes (CRA-PP, Pobè, Benin), for their logistical support and assistance during the collection of the oil palm plant material used in this study. We would like to thank Professor Reidunn B. Aalen for providing the IDA peptides used in the experiments. We also greatly appreciate the assistance of Stéphane Dussert for the statistical analysis of the oil palm experiments.

Conflicts of Interest: The authors declare no conflict of interest.

\section{References}

1. Roberts, J.A.; Elliott, K.A.; Gonzalez-Carranza, Z.H. Abscission, dehiscence, and other cell separation processes. Annu. Rev. Plant Biol. 2002, 53, 131-158. [CrossRef] [PubMed]

2. Estornell, L.H.; Agusti, J.; Merelo, P.; Talon, M.; Tadeo, F.R. Elucidating mechanisms underlying organ abscission. Plant Sci. 2013, 199-200, 48-60. [CrossRef]

3. Addicott, F.T. Abscission; University of California Press: Berkeley, CA, USA; Los Angeles, CA, USA, 1982.

4. Bleecker, A.B.; Patterson, S.E. Last exit: Senescence, abscission, and meristem arrest in Arabidopsis. Plant Cell 1997, 9, 1169-1179. [CrossRef] [PubMed]

5. Patterson, S.E. Cutting loose. Abscission and dehiscence in Arabidopsis. Plant Physiol. 2001, 126, 494-500. [CrossRef]

6. Patterson, S.E.; Butenko, M.A.; Kim, J. Ethylene responses in abscission and other processes of cell separation in Arabidopsis. In Advances in Plant Ethylene Research; Ramina, A., Chang, C., Giovannoni, J., Klee, H., Perata, P., Woltering, E., Eds.; Springer: Dordrecht, The Netherlands, 2007; pp. 271-278.

7. Patharkar, O.R.; Walker, J.C. Core Mechanisms Regulating Developmentally Timed and Environmentally Triggered Abscission. Plant Physiol. 2016, 172, 510-520. [CrossRef]

8. Patharkar, O.R.; Walker, J.C. Advances in abscission signaling. J. Exp. Bot. 2018, 69, 733-740. [CrossRef] [PubMed]

9. Patterson, S.E.; Bolivar-Medina, J.L.; Falbel, T.G.; Hedtcke, J.L.; Nevarez-McBride, D.; Maule, A.F.; Zalapa, J.E. Are We on the Right Track: Can Our Understanding of Abscission in Model Systems Promote or Derail Making Improvements in Less Studied Crops? Front. Plant Sci. 2016, 6, 1268. [CrossRef] [PubMed]

10. Meir, S.; Philosoph-Hadas, S.; Riov, J.; Tucker, M.L.; Patterson, S.E.; Roberts, J.A. Re-evaluation of the ethylene-dependent and -independent pathways in the regulation of floral and organ abscission. J. Exp. Bot. 2019, 70, 1461-1467. [CrossRef]

11. Butenko, M.A.; Patterson, S.E.; Grini, P.E.; Stenvik, G.E.; Amundsen, S.S.; Mandal, A.; Aalen, R.B. Inflorescence deficient in abscission controls floral organ abscission in Arabidopsis and identifies a novel family of putative ligands in plants. Plant Cell 2003, 15, 2296-2307. [CrossRef] [PubMed]

12. Stenvik, G.E.; Tandstad, N.M.; Guo, Y.; Shi, C.L.; Kristiansen, W.; Holmgren, A.; Clark, S.E.; Aalen, R.B.; Butenko, M.A. The EPIP peptide of INFLORESCENCE DEFICIENT IN ABSCISSION is sufficient to induce abscission in arabidopsis through the receptor-like kinases HAESA and HAESA-LIKE2. Plant Cell 2008, 20, 1805-1817. [CrossRef]

13. Butenko, M.A.; Wildhagen, M.; Albert, M.; Jehle, A.; Kalbacher, H.; Aalen, R.B.; Felix, G. Tools and Strategies to Match Peptide-Ligand Receptor Pairs. Plant Cell 2014, 26, 1838-1847. [CrossRef]

14. Cho, S.K.; Larue, C.T.; Chevalier, D.; Wang, H.; Jinn, T.L.; Zhang, S.; Walker, J.C. Regulation of floral organ abscission in Arabidopsis thaliana. Proc. Natl. Acad. Sci. USA 2008, 105, 15629-15634. [CrossRef] [PubMed]

15. Estornell, L.H.; Wildhagen, M.; Perez-Amador, M.A.; Talon, M.; Tadeo, F.R.; Butenko, M.A. The IDA Peptide Controls Abscission in Arabidopsis and Citrus. Front. Plant Sci. 2015, 6, 1003. [CrossRef] [PubMed] 
16. Ying, P.Y.; Li, C.Q.; Liu, X.C.; Xia, R.; Zhao, M.L.; Li, J.G. Identification and molecular characterization of an IDA-like gene from litchi, LcIDL1, whose ectopic expression promotes floral organ abscission in Arabidopsis. Sci. Rep.-UK 2016, 6, 37135. [CrossRef]

17. Stø, I.M.; Orr, R.J.S.; Fooyontphanich, K.; Jin, X.; Knutsen, J.M.B.; Fischer, U.; Tranbarger, T.J.; Nordal, I.; Aalen, R.B. Conservation of the abscission signaling peptide IDA during Angiosperm evolution: Withstanding genome duplications and gain and loss of the receptors HAE/HSL2. Front. Plant Sci. 2015, 6, 931. [CrossRef] [PubMed]

18. Jin, X.; Zimmermann, J.; Polle, A.; Fischer, U. Auxin is a long-range signal that acts independently of ethylene signaling on leaf abscission in Populus. Front. Plant Sci. 2015, 6, 634. [CrossRef]

19. Henderson, J.; Osborne, D.J. Cell Separation and Anatomy of Abscission in the Oil Palm, Elaeis guineensis Jacq. J. Exp. Bot. 1990, 41, 203-210. [CrossRef]

20. Osborne, D.J.; Henderson, J.; Corley, R.H.V. Controlling Fruit Shedding in the Oil Palm. Endeavour 1992, 16, 173-177. [CrossRef]

21. Roongsattham, P.; Morcillo, F.; Jantasuriyarat, C.; Pizot, M.; Moussu, S.; Jayaweera, D.; Collin, M.; Gonzalez-Carranza, Z.; Amblard, P.; Tregear, J.W.; et al. Temporal and spatial expression of polygalacturonase gene family members reveals divergent regulation during fleshy fruit ripening and abscission in the monocot species oil palm. BMC Plant Biol. 2012, 12, 150. [CrossRef] [PubMed]

22. Roongsattham, P.; Morcillo, F.; Fooyontphanich, K.; Jantasuriyarat, C.; Tragoonrung, S.; Amblard, P.; Collin, M.; Mouille, G.; Verdeil, J.L.; Tranbarger, T.J. Cellular and Pectin Dynamics during Abscission Zone Development and Ripe Fruit Abscission of the Monocot Oil Palm. Front. Plant Sci. 2016, 7, 540. [CrossRef] [PubMed]

23. Fooyontphanich, K.; Morcillo, F.; Amblard, P.; Collin, M.; Jantasuriyarat, C.; Tangphatsornruang, S.; Verdeil, J.L.; Tranbarger, T.J. A phenotypic test for delay of abscission and non-abscission oil palm fruit and validation by abscission marker gene expression analysis. Acta Hortic. 2016, 1119, 97-104. [CrossRef]

24. Tisné, S.; Denis, M.; Domonhédo, H.; Pallas, B.; Cazemajor, M.; Tranbarger, T.J.; Morcillo, F. Environmental and trophic determinisms of fruit abscission in African oil palm and climate change perspective. New Phytologist. submitted.

25. Tranbarger, T.J.; Dussert, S.; Joet, T.; Argout, X.; Summo, M.; Champion, A.; Cros, D.; Omore, A.; Nouy, B.; Morcillo, F. Regulatory mechanisms underlying oil palm fruit mesocarp maturation, ripening, and functional specialization in lipid and carotenoid metabolism. Plant Physiol. 2011, 156, 564-584. [CrossRef] [PubMed]

26. Jinn, T.L.; Stone, J.M.; Walker, J.C. HAESA, an Arabidopsis leucine-rich repeat receptor kinase, controls floral organ abscission. Genes Dev. 2000, 14, 108-117. [CrossRef]

27. Santiago, J.; Brandt, B.; Wildhagen, M.; Hohmann, U.; Hothorn, L.A.; Butenko, M.A.; Hothorn, M. Mechanistic insight into a peptide hormone signaling complex mediating floral organ abscission. Elife 2016, 5. [CrossRef]

28. Stenvik, G.E.; Butenko, M.A.; Urbanowicz, B.R.; Rose, J.K.; Aalen, R.B. Overexpression of INFLORESCENCE DEFICIENT IN ABSCISSION activates cell separation in vestigial abscission zones in Arabidopsis. Plant Cell 2006, 18, 1467-1476. [CrossRef]

29. Wilmowicz, E.; Kućko, A.; Ostrowski, M.; Panek, K. INFLORESCENCE DEFICIENT IN ABSCISSION-like is an abscission-associated and phytohormone-regulated gene in flower separation of Lupinus luteus. Plant Growth Regul. 2018, 85, 91-100. [CrossRef]

30. Henderson, J.; Osborne, D.J. Intertissue Signaling during the 2-Phase Abscission in Oil Palm Fruit. J. Exp. Bot. 1994, 45, 943-951. [CrossRef]

31. Newman, D. The distribution of range in samples from a normal population, expressed in terms of an independent estimate of standard deviation. Biometrika 1939, 31, 20-30. [CrossRef]

32. Keuls, M. The use of the "studentized range" in connection with an analysis of variance. Euphytica 1952, 1, 112-122. [CrossRef]

(C) 2019 by the authors. Licensee MDPI, Basel, Switzerland. This article is an open access article distributed under the terms and conditions of the Creative Commons Attribution (CC BY) license (http://creativecommons.org/licenses/by/4.0/). 